\title{
丙烯腈催化二聚反应的研究进展
}

\author{
俞 磊*,a,b,c 王 俊 ${ }^{a}$ 曹洪恩 ${ }^{a}$ 丁克鸿 ${ }^{a, b, c}$ 徐 清*,a,b,c \\ ( ${ }^{a}$ 江苏省环境材料与环境工程重点实验室 扬州大学化学化工学院 扬州 225002) \\ ( ${ }^{b}$ 温州大学化学与材料工程学院 温州 325035) \\ ( ${ }^{c}$ 江苏扬农化工集团有限公司 扬州 225009)
}

\begin{abstract}
摘要 丙烯腈是廉价易得的化工原料. 丙烯腈的线性二聚可以构建己(烯)二腈分子骨架，经过进一步的加氢还原可制 备己二胺. 己二胺是重要的工业中间体, 有着广泛的用途和广阔的市场, 主要用于合成尼龙 66. 因此, 丙烯腈的线性二 聚是非常重要的有机化学反应. 与已经实现工业化的电解法相比, 催化二聚有着能耗低、装置要求低等优点. 除此之 外，丙烯腈催化二聚还可能生成非线性聚合产物 2-亚甲基戊二腈. 它是生产广谱抗菌剂溴菌腈的重要中间体. 溴菌腈 的市场需求量虽然不如尼龙 66 巨大, 但也是重要的化工产品之一. 然而, 在丙烯腈的催化二聚领域, 至今未有系统性 的综述报导. 对丙烯腈催化二聚反应的研究进展进行整理与系统性地阐述，按照催化剂种类，主要分为钉催化、其它金 属催化以及膦催化二聚等三部分，供国内相关领域技术人员参考.
\end{abstract}

关键词 丙烯腈; 二聚; 催化; 己二腈; 己二胺; 高聚物; 尼龙 66; 溴菌腈

\section{A Review on Catalyzed Dimerization of Acrylonitrile}

\author{
Yu, Lei ${ }^{*, a, b, c} \quad$ Wang, Jun $^{a} \quad$ Cao, Hongen ${ }^{a} \quad$ Ding, Kehong $^{a, b, c} \quad$ Xu, Qing ${ }^{*, a, b, c}$ \\ ( ${ }^{a}$ Jiangsu Key Laboratory of Environmental Material and Environmental Engineering, School of Chemistry and \\ Chemical Engineering, Yangzhou University, Yangzhou 225002) \\ ( ${ }^{b}$ College of Chemistry and Materials Engineering, Wenzhou University, Wenzhou 325035) \\ ( ${ }^{c}$ Jiangsu Yangnong Chemical Engineering Co. Ltd., Yangzhou 225009)
}

\begin{abstract}
Acrylonitrile (AN) is a cheap and accessible industrial material. Linear dimerization of acrylonitrile affords the skeleton of adiponitrile, which leads to hexamethylene diamine through further hydrogenation. Hexamethylene diamine is an important industrial intermediate with comprehensive applications and wide market requirement and is mainly applied in the synthesis of nylon 66. Therefore, linear dimerization of acrylonitrile is a very important organic reaction. Compared with the electrolytic process which has already been applied in industrial production, the catalyzed dimerization needs low energy consumption and low equipment requirements. Besides, the catalyzed dimerization of acrylonitrile may also afford the branched dimer 2-methyleneglutaronitrile, which is the important intermediate in the synthesis of bromothalonil. Although the market requirement of bromothalonil is not as huge as nylon 66, it is also one of the important chemical industrial products. However, currently, there was no systematic review on AN dimerization. Therefore, this review aims to reorganize and systematically describe the advances of catalyzed dimerization of acrylonitrile for reference of the related technicians in China, including the Ru-catalyzed dimerization, other metal-catalyzed dimerization and phosphorus-catalyzed dimerization.
\end{abstract}

Keywords acrylonitrile; dimerization; catalysis; adiponitrile; hexamethylene diamine; polymer; nylon 66; bromothalonil

己二胺是重要的化工生产中间体，主要用于生产尼 龙 66. 尼龙 66 是最早实现工业化的聚酰胺, 是最重要的
两大聚酰胺品种之一．己二胺还用于制造其他种类聚酰 胺如尼龙 610 、尼龙 612 等，也是聚亚胺羧酸酯泡沫塑料

\footnotetext{
*E-mail: yulei@yzu.edu.cn; yzuyulei@163.com

Received May 4, 2014; revised May 21, 2014; published online June 11, 2014

Project supported by the National Natural Science Fundation of China (No. 21202141), the Priority Academic Program Development of Jiangsu Higher Education Institutions, the Opening Foundation of the Key Laboratory of Environmental Materials and Engineering of Jiangsu Province (Nos. K100027, K090030) and the Opening Foundation of the Key Laboratory of Green Pesticide and Agricultural Bioengineering, Ministry of Education, Guizhou University (No. 2010GDGP0106).

国家自然科学基金(No. 21202141)、江苏省高校优势学科、江苏省环境材料与工程重点实验室开放基金(Nos. K100027, K090030)及贵州大学教育部绿 色农药与农业生物工程重点实验室开放基金(No. 2010GDGP0106)资助项目.
} 
和聚氨酯泡沫塑料、涂料的主要原料, 还可用作环氧树脂 的固化剂、有机交联剂、农药、铁矿和铜矿的浮选剂等. 因 此, 已二胺合成技术的开发一直是有机合成化学研究中 的重要课题之一. 目前, 合成已二胺的方法有多种, 主要 包括己二腈法、己二酸法、己内酰胺法、已二醇法. 其中, 己二腈法反应条件较温和, 所需温度压力较低, 故装置 较为安全. 目前, 绝大多数己二胺生产装置都采用己二 腈法, 即通过己二腈加氢还原生产己二胺.

丙烯腈(Acrylonitrile，简写作 AN)是廉价易得的工 业原料, 通过丙烯腈的线性二聚即可得到己(烯)二腈分 子骨架，进一步催化加氢可制备己二胺. 该合成路线原 料易得, 原子经济性高, 因此, 是合成已二腈以及进一 步合成已二胺最有潜力的生产路线(Scheme 1). 通过电 解法, 可实现丙烯腈的线性二聚, 从而进一步合成己二 胺 $^{[1]}$. 这一方法目前较成熟, 已实现工业化. 然而, 该方 法能耗大, 对设备投资要求高. 因此, 开发更加优化的 丙烯腈线性二聚方法, 不仅有着较好的学术意义, 同时 还有着很高的应用价值. 过渡金属催化反应自从 20 世 纪 70 年代兴起以来, 一直受到有机合成化学家们的青 睐. 过渡金属催化反应有着高选择性、高效率以及条件 温和等优点, 可以广泛应用于有机合成、药物合成以及 工业生产中. 在对丙烯腈二聚反应的研究中, 人们已经 开发出一系列过渡金属催化的丙烯腈二聚反应，从而为 合成已二胺提供了一系列可供选择的备用途径. 除了过 渡金属之外，一些膦化合物也被发现可催化丙烯腈二 聚, 从而能提供更加环保的可能的合成途径. 然而, 由 于丙烯腈二聚催化剂体系种类繁多, 聚合机理、方式亦 多种多样, 至今, 尚无专门综述对此进行系统性的总结, 相关评述性介绍较为分散 ${ }^{[2]}$. 因此, 本综述拟对丙烯腈 催化二聚反应做一个系统性的总结与展望, 供此领域内 的研究者参考.

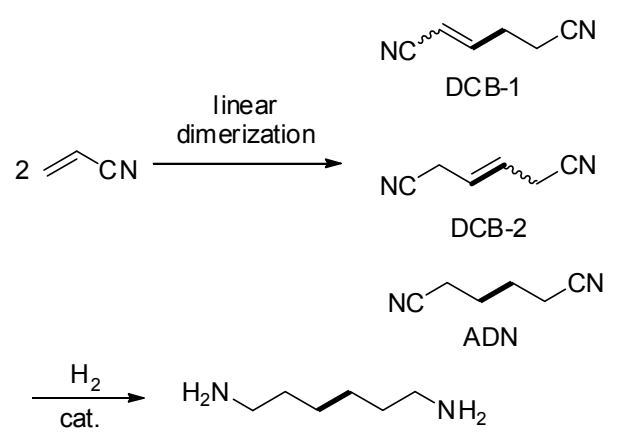

Scheme 1

\section{1 钉催化的丙烯腈二聚反应}

钓 (ruthenium, $\mathrm{Ru}$ ) 是一种硬而脆呈浅灰色的多价贵 金属元素, 是铂族金属中的一员, 被广泛应用于工业催 化中. 然而, 与铑、铂、钯、铱等贵金属相比, 钉的价格
相当便宜(图 1) ${ }^{[3]}$. 因此, 钓催化剂与其他贵金属催化剂 相比，有着显著的成本优势.

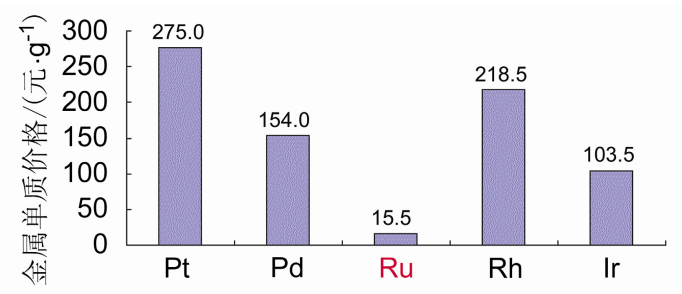

图 22013 年 12 月 20 日国内贵金属价格行情 Figure 2 Prices of expensive metals on Dec $20^{\text {th }}, 2013$ in China

在丙烯腈催化二聚反应中，钉催化的反应报道得最 多. 早在 1967 年, Misono 等 ${ }^{[4]}$ 即发现在氢气氛围下, 醇 溶剂中, 丙烯腈与三水合氯化钉反应, 可转化为丙腈 (PN)与二聚物 1,4-二腈基-1-丁烯(DCB-1) (Eq. 1).

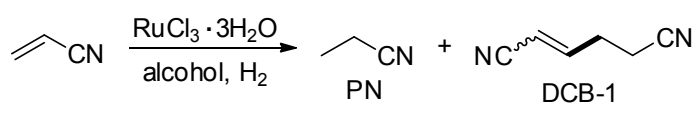

为了阐明该反应的机理, 他们研究了三水合氯化钉 与丙烯腈在乙醇溶剂中回流或 $150{ }^{\circ} \mathrm{C}$ 下的反应，分别 得到两种黄色复合物. 这两种物质与前一年所报道的丙 烯腈一氯化铑复合物在结构上和化学性质上有着显著的 区别 ${ }^{[5]}$. 其中, 在乙醇中回流制备的复合物易溶于二氯 甲烷和氯仿，溶于甲醇和乙醇，但几乎不溶于丙酮与乙 醚, 在真空中 $100{ }^{\circ} \mathrm{C}$ 以上即分解释放出丙烯腈. 元素分 析表明该物质的分子式为 $\left(\mathrm{CH}_{2}=\mathrm{CHCN}\right)_{3}-\mathrm{RuCl}_{2}$, 红外 波谱分析研究及文献[6]表明该物质通过丙烯腈氮上的 孤对电子与金属钉络合而成. 该物质可催化丙烯腈的二 聚反应. 在 $150{ }^{\circ} \mathrm{C}$, 氢气氛围中反应 $1 \mathrm{~h}$, 丙烯腈可被转 化为丙腈 $(\mathrm{PN}, 46 \%)$ 、已二腈( $\mathrm{ADN}, 4 \%$ )以及 1,4 二腈基1-丁烯(DCB-1, 50\%) (Eq. 2). 而三水合氯化钉与丙烯腈 在乙醇中 $150{ }^{\circ} \mathrm{C}$ 下反应得到的另一种复合物, 亦可催 化丙烯腈的二聚反应. 该络合物真空中加热到 $100{ }^{\circ} \mathrm{C}$ 即释放出丙腈和丙烯腈混合物，表明该物质中存在金属 与腈乙基结合的结构.

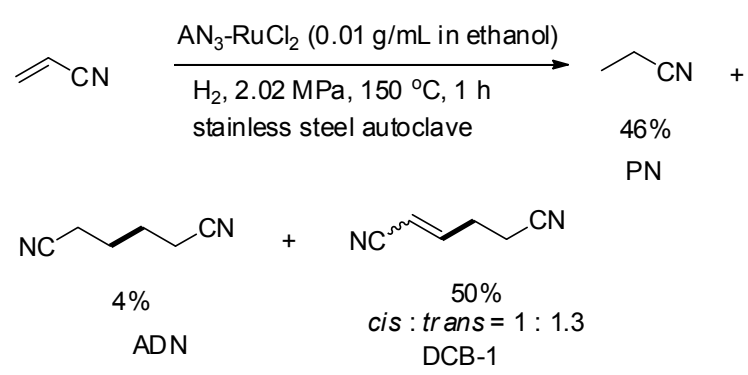

该工作是钉化合物催化丙烯腈的线性二聚以构建 己二腈分子骨架的最早报导. 第二年，他们进一步研究 
了氯化钓催化丙烯腈的二聚反应 ${ }^{[7]}$. 研究表明, 在三水 合氯化钓催化下, $3.03 \mathrm{MPa}$ 氢气氛围中, $150{ }^{\circ} \mathrm{C}$ 反应效 果最佳, 反应 $0.5 \mathrm{~h}$, 丙烯腈转化率可达 $94 \%$, 分别以 $51 \%$ 和 $44 \%$ 的产率生成丙腈与二聚物. 作为对比, 他们 还尝试了其它金属氯化物作为催化剂, 例如 $\mathrm{RhCl}_{3} \cdot 3 \mathrm{H}_{2} \mathrm{O}, \mathrm{MoCl}_{5}, \mathrm{WCl}_{6}, \mathrm{IrCl}_{4}, \mathrm{PdCl}_{2}$ 等. 然而, 研究 结果表明, 在这些金属催化下, 仅能观察到氢化产物丙 腈，完全没有二聚物生成.上述实验结果表明，金属钉 在丙烯腈二聚反应中有着独特的催化作用. 在后续研究 中, 他们尝试通过加入添加物如 $\mathrm{SnCl}_{2} \cdot 2 \mathrm{H}_{2} \mathrm{O}, \mathrm{LiCl}, \mathrm{KCl}$, $\mathrm{LiBr}, \mathrm{NaI}$, 吡啶, 苯并吡啶等来改进反应, 然而这些尝 试并不成功, 二聚物的产率并未通过添加这些物质而得 以提高.

随后, 他们进一步研究了钉复合物催化丙烯腈二聚 反应 ${ }^{[8]}$. 在这部分工作中, 他们检验了一系列钉复合物 如 $\mathrm{RuCl}_{2}\left(\mathrm{C}_{12} \mathrm{H}_{18}\right), \mathrm{Ru}(\mathrm{acac})_{3}, \mathrm{RuCl}_{2}(\mathrm{CO})_{2}(\mathrm{py})_{2}, \mathrm{RuCl}_{2}$ $\left(\mathrm{P}(\mathrm{OPh})_{3}\right)_{4}, \mathrm{RuCl}_{2}\left(\mathrm{PPh}_{3}\right)_{4}, \mathrm{RuCl}_{2}\left(\mathrm{PPh}_{3}\right)_{2}(\mathrm{an})_{2}, \mathrm{Ru}(\mathrm{acac})_{2}-$ $\left(\mathrm{PPh}_{3}\right)_{2}$ 的催化活性. 研究结果表明 $\mathrm{Ru}(\mathrm{acac})_{3}$ 催化效果 最佳, 其中, 丙烯腈转化率可达 98.2\%、氢化还原产物 丙腈的产率为 $39.6 \%$, 而线性二聚产物 (cis 或 trans DCB-1 以及己二腈)总产率可达 $60.9 \%$, 反应的 TON 约 为 118 .

几乎在同一时间, McClure 等 ${ }^{[9]}$ 也独立地发现了钉 催化的丙烯腈二聚反应. 通过一系列更详细的机理研究 实验，他们提出了该二聚反应可能的机理(Scheme 2). 首先在氢气氛围中, 钉催化剂 $\mathbf{1}$ 可被氢化还原为钉氢化 物 $\mathbf{2}^{[10]}$. 该反应会释放出一分子氯化氢, 因此, 碱的存 在能够促进该反应进行. 钓氢化物 2 中的钉-氢键较活 泼, 被插入一分子丙烯腈后, 可生成中间体 3. 中间体 $\mathbf{3}$ 的钓一碳 $\sigma$ 键亦较活泼, 可进一步被另一分子丙烯腈双 键插入, 从而生成中间体 4. 进一步的 $\beta$-氢消除, 可生 成线性二聚产物 DCB-1 并重新形成活性催化物种 2. 不 难看出, 该反应的关键是生成活性催化剂物种 2. 而生 成 2 的关键是要有氢气存在, 这也正是该反应在氮气中 无法进行的原因. 然而, 氢气能够直接还原中间体 $\mathbf{3}$, 生成丙腈(Eq. 3). 因此, 该反应中不可避免有相当量的 副产物丙腈产生. 15 年后, Tsou 等 ${ }^{[11]}$ 通过同位素気标记 实验, 进行了更详细的机理研究. 实验结果表明, 当反 应在氛氛围中进行时, 产物 DCB-1 中与腈基相邻的亚 甲基氛代(CHD)的比例高达 39.3\% ${ }^{[12]}$. 两年后, 他们还 进行了动力学研究实验 ${ }^{[13]}$. 这些实验结果使得该反应 机理更加明确. 1998 年, Fukuoka 等 ${ }^{[14]}$ 对这一反应进行 了进一步的优化. 他们发现, 在无溶剂条件下, 使用低 价钉作催化剂, 可以显著优化丙烯腈的线性二聚反应. 该反应的 TON 最高可提高到 2000 以上, 线性二聚产物
总产率可达 42\%，但无法避免地，仍然有 19\%的副产物 丙腈生成 ${ }^{[14]}$.

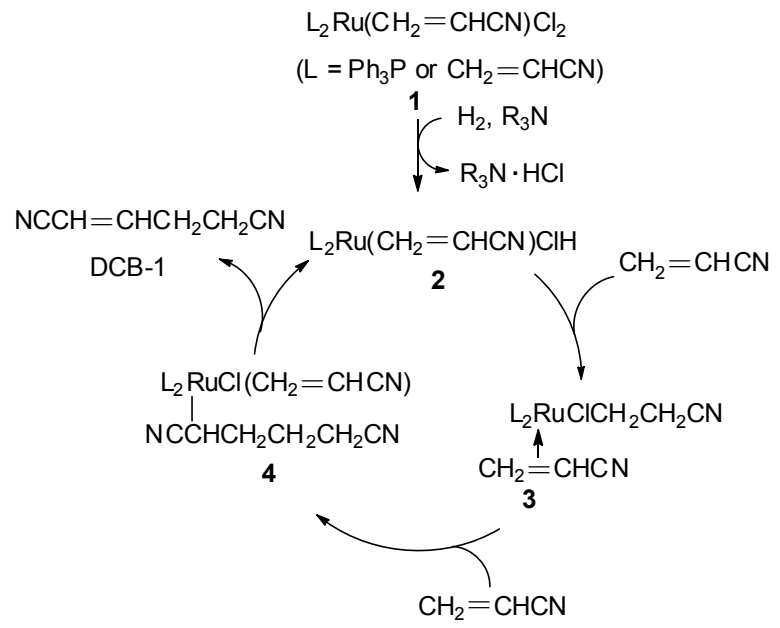

Scheme 2

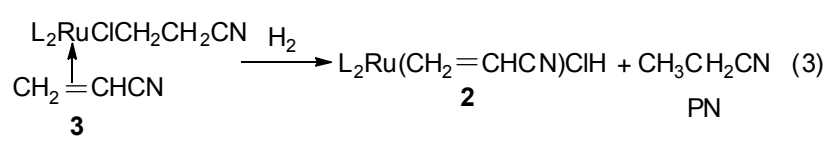

氢气氛围中的丙烯腈催化线性二聚反应，可构建己 二腈分子骨架，并且该反应可通过选择适合的配体与反 应条件来加以调控，提高产率和 TON，但却不可避免地 会有丙腈生成，从而降低了原料利用率，难以应用到工 业生产. 因此, 之后数十年, 人们一直在尝试开发避免 丙腈副产物生成的催化体系. 1997 年, Kashiwagi 等 ${ }^{[15]}$ 报 道了一个新的催化剂体系, 即 $\mathrm{RuCl}_{2}(\mathrm{DMSO})_{4} /$ $\mathrm{CH}_{3} \mathrm{CH}_{2} \mathrm{COONa} / \mathrm{DMSO} /$ 羧酸催化剂体系. 利用这一催 化剂体系，反应无需在氢气氛围下进行，因此也不会生 成还原产物丙腈, 大大提高了原料利用率. 通过引入羧 酸，反应中生成的钓中间体可发生酸解，从而生成 DCB-1 并进一步实现催化剂再生. 该反应详细机理如下 所示(Scheme 3): 钓催化剂首先插入丙烯腈末端的 C$\mathrm{H}$ 键，形成中间体 5. 中间体 5 的 $\mathrm{C}-\mathrm{Ru}$ 键进一步被另 一分子丙烯腈插入, 形成与 Scheme 3 中 4 结构类似的中 间体 6. 在羧酸作用下, 6 可以发生酸解, 生成产物 DCB- 1 和金属中间体物种 7. 7 通过还原消除脱去羧酸可 重新生成催化剂. 数年后, 他们进一步研究了羧酸酸性 强度与反应之间的关系. 研究表明, $\mathrm{p} K_{\mathrm{a}} 3.5 \sim 5.0$ 之间 的羒酸是最佳的助催化剂. 而二聚物生成速率的对数函 数与 $\mathrm{p} K_{\mathrm{a}}$ 之间呈线性关系. 这一发现为今后进一步优化 该反应，篮选适当的羧酸，奠定了理论基础 ${ }^{[16]}$. 随后, 他们进行了更细致的研究，检验了该反应在不同条件下 的结果, 发现二苯醚是最佳溶剂. 在此条件下, 反应 TON 值最高可调节到近 $1800^{[17]}$. 然而, 虽然通过酸解 机理进行的催化二聚反应可以完全抑制丙腈和非线性 
二聚物的生成, 并且进一步更加细致的工作将线性二聚 物的选择性提高到 85.8 , 反应物丙烯腈也可作溶剂, 大 大提高了该反应在工业生产中应用的可行性 ${ }^{[18]}$, 该反 应仍然存在着一些问题. 除了原料转化率不高之外, 最 重要的问题在于该反应的催化剂体系过于复杂, 添加物 过多, 除了催化剂、配体、助催化剂羧酸与羧酸钠之外, 为了抑制羧酸与丙烯腈的加成反应, 还需添加羧酸腈乙 基酯 ${ }^{[18]}$. 这些添加物在反应后难以回收利用, 在工业生 产中将形成大量的废弃物, 而不符合绿色化学精神. 添 加物的过多还会导致催化剂失活. 例如, 作者在随后的 研究中发现, 作为配体的 DMSO 与作为助催化剂的羧 酸反应, 生成的甲硫甲基羧酸酯, 会毒化催化剂 ${ }^{[19]}$.

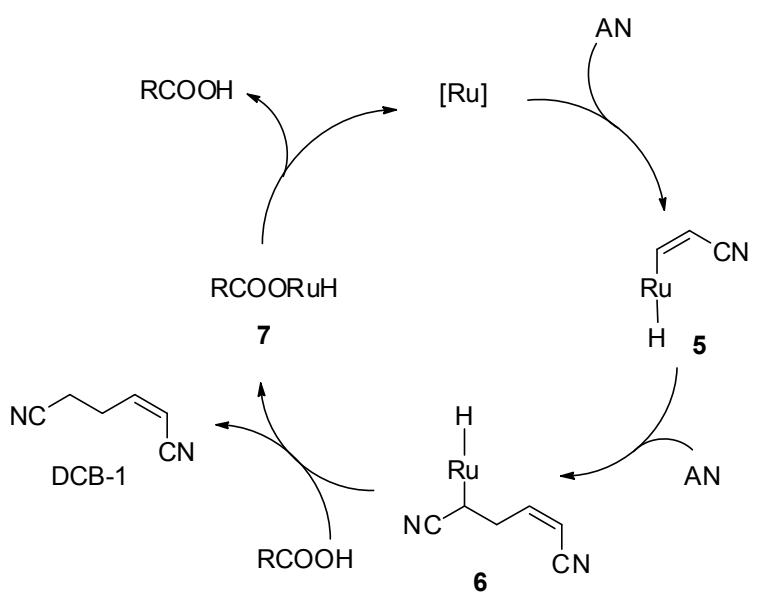

Scheme 3

综上所述, 现阶段, 钓催化丙烯腈线性二聚反应主 要有两种催化模式, 一种是通过氢解机理进行的 ${ }^{[4 \sim 14,20]}$, 另一种是通过酸解机理进行的 ${ }^{[15 ~ 19,21]}$. 这两种方法都存 在着明显的局限而尚不能在工业生产上应用. 前者不可 避免地会生成副产物丙腈, 线性二聚物选择性低, 原料 利用率不高. 而后者催化剂体系过于复杂, 反应结束后 会产生大量不可回收的废弃物, 不符合绿色化学精神. 因此, 开发更加简单高效的催化剂体系, 实现丙烯腈的 线性二聚, 仍然是有着很大的理论意义和应用价值的挑 战性课题, 也是我们团队正在努力的方向.

\section{2 其它金属催化的丙烯腈二聚反应}

虽然钉是贵金属之中较便宜的一种, 但其价格仍然 很高. 如果能够开发出普通金属催化丙烯腈二聚的反 应，显著降低催化剂成本，则应用价值更高，技术实现 工业化生产的可能性也更大. 因此, 人们从没有放弃过 开发其它廉价金属催化丙烯腈二聚的反应的努力.

早在 20 世纪 60 年代, Chiusoli 小组 ${ }^{[22]}$ 即报道了一则 利用等物质的量的的氯化钴和锰单质, 促进丙烯腈线性
聚合的反应(Eq. 4). 他们将 $50 \mathrm{mmol}$ 锰粉、 $30 \mathrm{mmol}$ 氯 化钴、 $60 \mathrm{mmol}$ 丙烯腈(含有对苯二酚稳定剂)在 $50 \mathrm{~mL}$ DMF 中室温 $\left(20^{\circ} \mathrm{C}\right)$ 振摇 $20 \mathrm{~h}$ 后, 滤去未反应的锰粉并 通入硫化氢. 通过蒸馏可得到 $29 \mathrm{mmol}$ 己二腈(ADN, 含有 $0.1 \%$ 的非线性二聚产物 2-亚甲基戊二腈). 该反应 产率极高, 并且成本不高, 甚至要低于前述钓催化剂催 化的技术. 然而, 该反应会产生大量固体废弃物. 在环 境保护呼声越来越高的今天，这种技术难以实现工业 化. 但是, 该反应仍然可以给我们启发: 如果能够开发 新型高效催化剂, 将还原剂由锰替换为更加清洁的物质 (例如氢气), 类似技术将来或许有实现工业化的可能. 这也可能是今后新技术开发的发展方向.

$$
\begin{aligned}
& 2 ح \mathrm{CN}+\mathrm{Mn}+\mathrm{CoCl}_{2}+2 \mathrm{H}_{2} \mathrm{O} \underset{20 \mathrm{~h}}{\stackrel{20^{\circ} \mathrm{C}}{\longrightarrow}} \mathrm{NC} \underset{\mathrm{ADN}}{\mathrm{CN}}+ \\
& \text { 95\% (based on the } \\
& \text { converted AN) } \\
& \mathrm{MnCl}_{2}+\mathrm{Co}(\mathrm{OH})_{2}
\end{aligned}
$$

此外, 日本科学家还报道了铜、镍、钴、锌等廉价 金属催化丙烯腈二聚的反应 ${ }^{[23 ~ 25]}$. 然而与铑催化反应 不同, 这些廉价金属催化丙烯腈二聚, 并不能生成线性 的已二腈衍生物, 而是得到非线性的 2-亚甲基戊二腈 (Eq. 5). 虽然市场需求远不如已二腈衍生物巨大，2-亚 甲基戊二腈也是一种非常有用的化工中间体, 它与溴加 成，可以制备溴菌腈(Bromothalonil)，一种广谱、高效、 低毒的杀菌剂(Eq. 6). 在这一部分的研究中, Watanabe 与 Takeda ${ }^{[24]}$ 做了较详细的工作, 仔细篮选了一系列催 化剂, 并总结出一定规律. 其中, 对于金属来说, 催化 效果 $\mathrm{Co}(\mathrm{II}) \geqslant \mathrm{Zn}(\mathrm{II}) \gg \mathrm{Fe}(\mathrm{II})>\mathrm{Al}(\mathrm{III}) \gg \mathrm{V}(\mathrm{III})>\mathrm{Cd}(\mathrm{II})>$ $\mathrm{Ti}(\mathrm{IV})$; 而对于阴离子, 催化效果规律则是: $\mathrm{I}>\mathrm{Br}>\mathrm{Cl}$. 该反应中还需要添加胺类, 而三乙胺效果最 佳 $^{[24]}$. 随 后, Nomura 等 ${ }^{[25]}$ 还报道了镍催化丙烯腈非线性二聚合 成 2-亚甲基戊二腈的反应. 然而, 该反应需要添加膦配 体和卤代烃, 并且在含卤溶剂中进行, 转化率和选择性 都较低, 与前人工作相比, 并没有明显的优势. 并且, 鉴于我们课题组近期的研究成果, 我们怀疑该反应的真 正催化剂物种并非过渡金属, 而是反应中所添加的膦配 体(见下一节讨论).
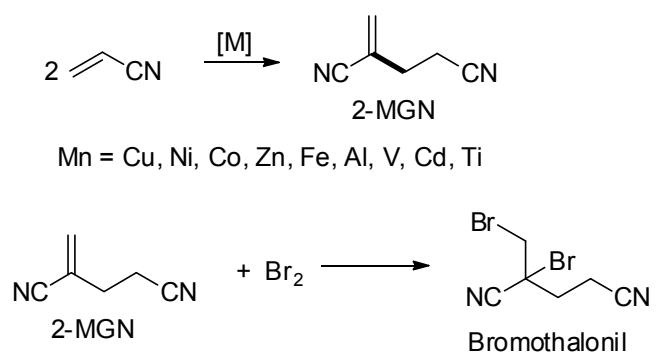


\section{3 膦催化的丙烯腈二聚反应}

在催化反应中，膦催化的有机合成反应独树一 帜 ${ }^{[26,27]}$. 膦催化的反应不使用金属，产物中不会有金属 残留, 从而更加适用于对金属残留量要求严格的药物合 成领域. 避免使用金属催化剂的合成策略对环境更加友 好. 因此, 膦催化的有机合成反应长期以来一直吸引着 人们的注意. 在丙烯腈催化二聚反应这一领域, 早在 20 世纪 60～70 年代, McClure ${ }^{[28]}$ 已经尝试用膦来催化二聚 (Eq. 7). 该反应需在高温 $\left(160 \sim 175{ }^{\circ} \mathrm{C}\right)$ 下进行, 虽然二 聚物总产率最高可达到 $90 \%$, 但产物中最重要的线性二 聚产物 DCB-1 所占比重较低，最高仅达到 $40 \%{ }^{[28]}$.

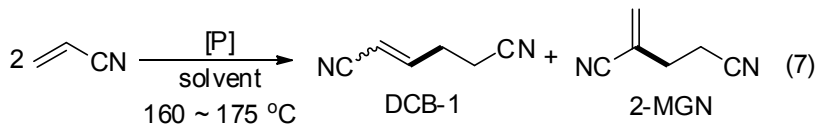

$[\mathrm{P}]=\mathrm{Ph}_{3} \mathrm{P}$ or $\left(p-\mathrm{CH}_{3} \mathrm{C}_{6} \mathrm{H}_{4}\right)_{3} \mathrm{P} \quad$ DCB-1 $+2-\mathrm{MGN}$ yield up to $90 \%$ solvent $=\mathrm{Me}_{3} \mathrm{COH}$ or $\mathrm{Et}_{3} \mathrm{SiOH} \quad$ DCB-1 selectivity up to $40 \%$

基于作者所进行的实验结果以及近年来有关膦催 化反应的文献报导 ${ }^{[22 ~ 24]}$, 该反应机理不难理解(Scheme 4): 首先, 由于带有孤对电子, 膦具有一定的亲核性, 可与丙烯腈发生加成反应, 生成中间体 8 . 中间体 8 上 的碳负离子可进一步与另一分子丙烯腈加成, 生成中间 体 9.9 发生氢转移, 可生成中间体 $\mathbf{1 0}$, 经过消除反应生 成非线性二聚产物 2-亚甲基戊二腈并实现催化剂膦的 再生. 以上是非线性二聚的机理. 而反应产物中同时还 观察到线性二聚产物 DCB-1. 该物质生成的关键, 在于 中间体 8 的氢转移(Scheme 5). 在质子试剂的作用下, 中间体 8 可发生氢转移而生成 11.11 与另一分子丙烯腈 加成可生成 12 , 通过氢转移而生成 13.13 经过消除反 应，可生成线性二聚产物 DCB-1 并实现膦催化剂的再 生(Scheme 5). 由于上述种种优点, 膦催化丙烯腈二聚 反应应用前景非常诱人. 因此, 20 世纪 90 年代, Jennings 小组 ${ }^{[29 ~ 31]}$ 对此展开了一系列的深入研究. 与 McClure 的 工作不同，他们以芳基次亚磷酸酯与亚磷酸酯作催化 剂, 从而使得反应可在温和条件下进行, 并且产物中线 性二聚产物的比例可提高到 $90 \%$ 以上. 通过一系列详细 的条件探索实验, 他们最终得出几个结论: (1)芳基磷酸 或次磷酸酯能够很好地催化丙烯腈线性二聚; (2)芳基亚 磷酸或次亚磷酸芳基对位含有推电子基团能够提高二 聚反应产率; (3)反应中必须要有醇, 否则丙烯腈会聚和; (4)膦催化剂体系对水和酚类稳定剂(丙烯腈中通常会添 加对苯二酚作为稳定剂)敏感, 会因此而失效; (5)提高反 应温度有利于提高二聚产率, 但超过 $60{ }^{\circ} \mathrm{C}$ 催化剂容易 失活; (6)亚磷酸或者次亚磷酸酯中的烷氧基对反应产率 与产物中线性二聚物比例影响甚微, 但会显著影响到催 化剂稳定性; (7)醇与丙烯腈加成的副产物可通过利用碳
氢溶剂稀释反应来避免，但代价是会降低二聚反应产 率. 这些结论对今后进一步设计膦催化剂催化丙烯腈二 聚反应提供了理论基础.

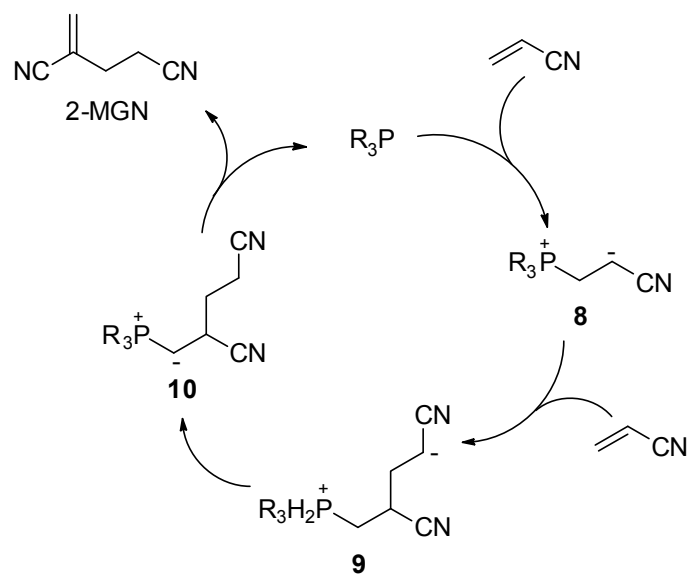

Scheme 4

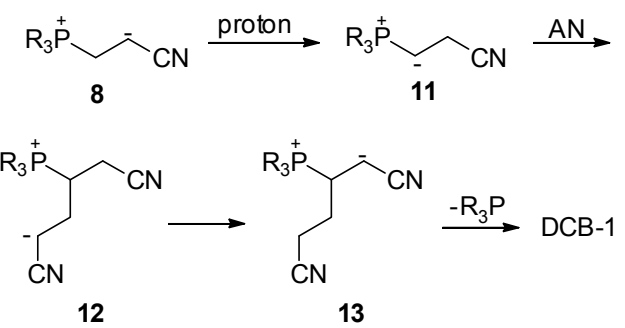

Scheme 5

最近，我们发现，三环己基膦可以催化丙烯腈二聚， 高选择性高产率地生成非线性聚合产物 2-亚甲基戊二 腈(Eq. 8) ${ }^{[32]}$. 与已知文献报道相比 ${ }^{[28,33]}$, 该反应在廉价 非卤溶剂叔丁醇中进行，反应条件温和，催化剂用量低， 并且反应浓度较高, 非常适合工业生产. 我们已经成功 将反应扩大到 $50 \mathrm{~mL}$ 丙烯腈规模. 该方法为生产农药中 间体 2-亚甲基戊二腈提供了高效清洁的合成方法.

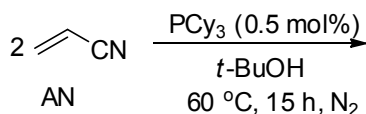

Starting material concentration $5 \mathrm{~mol} \cdot \mathrm{L}^{-1}$ Reaction scale up to $50 \mathrm{~mL} \mathrm{AN}$

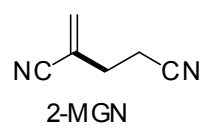

GC yield up to $90 \%$ Isolated yield up to $77 \%$

\section{4 结论与展望}

丙烯腈线性催化二聚制备已二腈衍生物从 20 世纪 60 年代开始就已经有人在研究, 目前催化方法主要分 为两种: 过渡金属催化 (主要是钉催化)与膦催化. 而过 渡金属钉催化的线性二聚又分为氢解催化与酸解催化 两种. 然而, 这些方法至今为止尚未足够完善以达到工 业生产的需求, 存在着或多或少的缺点, 例如转化率低, 
选择性低, 催化体系复杂且易失活, 产生大量废弃物等. 因此, 开发新一代高效清洁的新方法催化丙烯腈线性二 聚, 是今后工作的重点. 我们最近开发了较佳的以丙烯 腈非线性二聚制备 2-亚甲基戊二腈的合成方法，该方法 高效清洁、催化剂用量较小, 有望实现工业化应用的可 能. 目前我们正在为进一步放大反应做更加深入的研 究.

\section{References}

[1] Chem. Word 1963, 458 (in Chinese). (化学世界, 1963, 458.)

[2] Ciganek, E. Org. React. 1997, 51, 340.

[3] http://www.mining120.com/show/1312/ 20131220 130710.html

[4] Misono, A.; Uchida, Y.; Hidai, M.; Kanai, H. Chem. Commun. 1967, 357.

[5] Dewhirst, K. C. Inorg. Chem. 1966, 5, 319.

[6] Massey, A. G. J. Inorg. Nuclear Chem. 1962, 24, 1172.

[7] Misono, A.; Uchida, Y.; Hidai, M. Bull. Chem. Soc. Jpn. 1968, 41, 396.

[8] Misono, A.; Uchida, Y.; Hidai, M.; Inomata, I. Chem. Commun. 1968, 704.

[9] McClure, J. D.; Owyang, R.; Slaugh, L. H. J. Organomet. Chem. 1968, 12, P8.

[10] Hallman, P. S.; Evans, D.; Osborn, J. A.; Wilkinson, G. Chem. Commun. 1967, 305

[11] Tsou, D. T.; Burrington, J. D.; Maher, E. A.; Grasselli, R. K. J. Mol. Catal. 1983, 22, 29.

[12] As shown in Scheme 2, one of the $\mathrm{H}$ atom on the methylene came from hydrogen.

[13] Tsou, D. T.; Burrington, J. D.; Maher, E. A.; Grasselli, R. K. J. Mol. Catal. 1985, 30, 219.

[14] Fukuoka, A.; Nagano, T.; Furuta, S.; Yoshizawa, M.; Hirano, M.; Komiya, S. Bull. Chem. Soc. Jpn. 1998, 71, 1409.

[15] Kashiwagi, K.; Sugise, R.; Shimakawa, T.; Matuura, T.; Shirai, M.; Kakiuchi, F.; Murai, S. Organometallics 1997, 16, 2233.

[16] Kashiwagi, K.; Sugise, R.; Shimakawa, T.; Matuura, T.; Shirai, M. Chem. Lett. 2006, 35, 186.

[17] Kashiwagi, K.; Sugise, R.; Shimakawa, T.; Matuura, T. Chem. Lett. 2007, 36, 1384

[18] Kashiwagi, K.; Sugise, R.; Shimakawa, T.; Matuura, T.; Shirai, M. J. Mol. Catal. A: Chem. 2007, 264, 9.

[19] Kashiwagi, K.; Sugise, R.; Shimakawa, T.; Matuura, T.; Shirai, M. J. Mol. Catal. A: Chem. 2008, 286, 120.

[20] (a) Komiya, S.; Fukuoka, A.; Hirano, M. JP 09286769, 1997 [Chem. Abstr. 1997, 128, 3449].

(b) Suzuki, Y.; Kiso, Y. JP 9504307, 1997 [Chem. Abstr. 1997, 126, 157193].

(c) Murai, S.; Oodan, K.; Sugise, R.; Shirai, M.; Shimakawa, T. JP 06009531, 1994 [Chem. Abstr. 1994, 120, 191146].

[21] Sugise, R.; Shirai, M.; Shimakawa, T.; Kashiwagi, K. JP 05286918 , 1993 [Chem. Abstr. 1994, 120, 54210].

[22] Angès, G.; Chiusoli, G. P.; Cometti, G. Chem. Commun. 1968, 1515.

[23] Saegusa, T.; Ito, Y.; Kinoshita, H.; Tomita, S. Bull. Chem. Soc. Jpn. 1970, 43, 877 .

[24] Watanabe, Y.; Takeda, M. Bull. Chem. Soc. Jpn. 1973, 46, 883.

[25] Nomura, K.; Ishino, M. J. Mol. Catal. 1992, 73, L15.
[26] For reviews see: (a) Xie, P.-Z.; Huang, Y. Eur. J. Org. Chem. 2013, 6213.

(b) Xu, S.-L.; He, Z.-J. RSC Adv. 2013, 3, 16885.

(c) Wang, Y.-R.; Pan, J.-J.; Chen, Z.-D.; Sun, X.-Q.; Wang, Z.-M. Mini-Rev. Med. Chem. 2013, 13, 836.

(d) Wang, Z.-M.; Kwon, O. In Basics and Applications in Organic Synthesis, Drug Discovery, and Chemical Biology, Ed.: Trabocchi, A., Wiley-VCH, Hoboken, 2013, p. 97.

(e) Xu, S.-L.; He, Z.-J. Chin. J. Org. Chem. 2012, 32, 1159 (in Chinese).

(徐四龙, 贺峥杰, 有机化学, 2012, 32, 1159.)

(f) Methot, J. L.; Roush, W. R. Adv. Synth. Catal. 2004, 346, 1035.

[27] Selected recent articles: (a) Zheng, S.-Q.; Lu, X.-Y. Org. Lett. 2008, 10, 4481 .

(b) Lu, Z.; Zheng, S.-Q.; Zhang, X.-M.; Lu, X.-Y. Org. Lett. 2008, 10, 3267.

(c) Tian, J.-J.; Zhou, R.; Sun, H.-Y.; Song, H.-B.; He, Z.-J. J. Org. Chem. 2011, 76, 2374.

(d) Zhou, R.; Wang, J.-F.; Song, H.-B.; He, Z.-J. Org. Lett. 2011, 13, 580 .

(e) Cruz, D.; Wang, Z.-M.; Kibbie, J.; Modlin, R.; Kwon, O. Proc. Natl. Acad. Sci. U. S. A. 2011, 108, 6769.

(f) Wang, Z.-M.; Castellano, S.; Kinderman, S. S.; Argueta, C. E.; Beshir, A. B.; Fenteany, G.; Kwon, O. Chem. Eur. J. 2011, 17, 649. (g) Zhou, R.; Wang, J.-F.; Duan, C.; He, Z.-J. Org. Lett. 2012, 14, 6134.

(h) Zhou, R.; Wang, J.-F.; Tian, J.-J.; He, Z.-J. Org. Bioorg. Chem. 2012, 10, 773 .

(i) Zheng, J.; Huang, Y.; Li, Z.-M. Org. Lett. 2013, 15, 5064.

(j) Hu, C.-C.; Zhang, Q.-L.; Huang, Y. Chem. Asian J. 2013, 8, 1981.

(k) Li, E.-Q.; Huang, Y.; Liang, L.; Xie, P.-Z. Org. Lett. 2013, 15, 3138 .

(1) Zhou, R.; Wang, J.-F.; Yu, J.; He, Z.-J. J. Org. Chem. 2013, 78, 10596.

(m) Tian, J.-J.; He, Z.-J. Chem. Commun. 2013, 49, 2058.

[28] (a) McClure, J. D. J. Org. Chem. 1970, 35, 3045.

(b) McClure, J. D. US 3225083, 1965 [Chem. Abstr. 1966, 64, 103655].

[29] Jennings, J. R.; Cozens, R. J. Appl. Catal. A: Gen. 1995, 124, 297.

[30] Jennings, J. R.; Cozens, R. J.; Wade, K. Appl. Catal. A: Gen. 1995, $130,175$.

[31] Jennings, J. R.; Cozens, R. J. Appl. Catal. A: Gen. 1996, 135, 163.

[32] Yu, L.; Wang, J.; Zhang, X.; Cao, H.-E.; Wang, G.-L.; Ding, K.-H.; Xu, Q.; Lautens, M. RSC Adv. 2014, 4, 19122.

[33] (a) Charardes, P.; Grard, C.; Lafont P.; Thiers, M. FR 1366081, 1964 [Chem. Abstr. 1964, 61, 83787].

(b) Baizer, M. M.; Anderson, J. D. J. Org. Chem. 1965, 30, 1357.

(c) Jenner, G. Tetrahedron Lett. 2000, 41, 3091.

(d) Araki, Y.; Maki, T. JP 05001008, 1993 [Chem. Abstr. 1993, 118, 212465].

(e) Araki, Y.; Maki, T. JP 04368362, 1992 [Chem. Abstr. 1993, 118, 254412].

(f) Saito, M. JP 2012001521, 2012 [Chem. Abstr. 2012, 156, 122960].

(g) Ichikawa, S.; Iwane, H. JP 11180941, 1999 [Chem. Abstr. 1999, 131, 58603].

(h) Heckle, W. A., Mathews III, M. J.; Peoples, P. R. US 4952541, 1990 [Chem. Abstr. 1990, 113, 172961]. 